\title{
Quaderni
}

QUADERNI Communication, technologies, pouvoir

\section{Autorité et pratiques de légitimation en ligne}

\section{Étienne Candel et Pergia Gkouskou-Giannakou}

\section{(2) OpenEdition}

\section{Journals}

\section{Édition électronique}

URL : http://journals.openedition.org/quaderni/1066

DOI : 10.4000/quaderni.1066

ISSN : 2105-2956

\section{Éditeur}

Les éditions de la Maison des sciences de l'Homme

Édition imprimée

Date de publication : 5 mai 2017

Pagination : 5-11

\section{Référence électronique}

Étienne Candel et Pergia Gkouskou-Giannakou, « Autorité et pratiques de légitimation en ligne », Quaderni [En ligne], 93 | Printemps 2017, mis en ligne le 05 mai 2017, consulté le 24 septembre 2020. URL : http://journals.openedition.org/quaderni/1066 ; DOI : https://doi.org/10.4000/quaderni.1066 


\section{$D$ ossier}

\section{Autorité et pratiques de légitimation en ligne \\ Étienne Candel}

GRIPIC/Celsa Paris-Sorbonne

Pergia Gkouskou-Giannakou

GRIPIC/Université de la Nouvelle-Calédonie
Les espaces numériques ont de fraîche date été mobilisés, par des acteurs divers, comme lieux pour l'élaboration ou la transformation de postures et de relations d'autorité : encore aujourd'hui, les lieux de l'écriture en ligne voient se multiplier les pratiques sociales dans lesquelles la participation, la production de textes, la prise de parole dans des collectifs plus ou moins déterminés, plus ou moins définis, conditionnent des processus complexes d'évaluation et de reconnaissance. On sait par exemple la popularité de Wikipedia, qui en fait un lieu de référence de fait, ou la valeur que peut recouvrir, pour l'expression et la publication, le nombre de lecteurs réguliers d'un blog.

Différentes idéologies, différents imaginaires de la technique et des médias ont pu inspirer l'usage d'Internet dans ces visées de légitimation : l'apparition des notions de " communauté d'intérêt » (Licklider et Taylor, 1968), de « communauté en ligne » ou de « communauté virtuelle » (Rheingold, 1995) a pu par exemple s'exercer dans un contexte spécifique, celui du développement des réseaux parmi les chercheurs informaticiens des universités et grands centres de recherche américains, ou dans les groupes contre-culturels y voyant une façon de fonder un fonctionnement politique libertaire; commune à ces différentes théories, une pensée politique de l'écriture et de la publication, qui étaient censées permettre de se débarrasser des contingences - temps, localisation, statut social, caractéristiques physiques - au profit de la seule valorisation, de la seule reconnaissance des qualités intrinsèques d'un discours écrit, d'une pensée, d'une façon de voir. Sur Usenet ou sur les actuels forums spécialisés, la reconnaissance de la valeur 
d'un énoncé est censée relever de ses qualités propres ; elle serait l'expression pure, en quelque sorte, d'une légitimité « légitime», rationnelle, indépendante des héritages, des modes d'institution, des traditions et du nom propre.

Aiguë dans les milieux du développement informatique, où elle trouve une certaine effectivité, cette pensée de l'autorité fondée sur le seul travail du texte et sur le seul mérite dans le cadre d'un débat rationnel idéalisé, a largement diffusé son modèle dans les pratiques contemporaines, où le couple contribution-rétribution (Gomez-Mejia, 2016) semble sanctionner de façon exemplaire la relation entre la qualité d'un objet - un discours, une photographie, une blague - et ses effets de reconnaissance dans le public - les comptages discrets d'étoiles ou de pouces levés venant relayer les traditionnels applaudissements, le nombre de vues, le retour des visiteurs sur les lieux de la publication.

La question de la validité de ces conceptions n'est pas a priori pertinente : en soi, c'est plutôt leur opérativité symbolique qui importe. La pensée de l'autorité discursive, sur les réseaux, est ancrée dans un imaginaire vivace du numérique, dans une représentation partagée, qui inspire le travail des usagers. De sorte que le travail de l'autorité, ce processus social par lequel certains acteurs sont légitimes ou se légitiment, trouve dans les dispositifs spécifiques que sont les médias informatisés une valeur et une modalité particulière. Ces cadres sociaux voient se dérouler des phénomènes de légitimation, de reconnaissance, de partage et d'évaluation dont la portée imaginaire se réalise autant dans des discours que dans des pratiques.
L'objet du présent dossier est justement de permettre une appréhension des cadres médiatiques dans lesquels se déploient, pour les stratégies d'acteurs, les phénomènes communicationnels et les relations de pouvoir qui composent, socialement, la croyance partagée, la confiance, la valeur des discours et la position sociale de ceux qui les profèrent. La question politique qui détient une puissance sur le monde vécu ? - rencontre ici une question médiatique ou communicationnelle - où et selon quelles normes, quelles représentations, se construit cette autorité ? Ainsi, si l'on peut effectivement « faire autorité », et appeler à soi la croyance, la confiance ou la valeur sociale, cela ne se déroule jamais sans médiations, et en particulier sans médiations matérielles, concrètes, dans le cadre de dispositifs qui sont à la fois des objets d'énonciation - des énoncés, par exemple, de Twitter ou de Medium comme plateformes -, des objets fondant une énonciation - celle de leurs « membres »- et des objets utilisés par ces énonciateurs - dans le cadre d'une sociologie des usages mettant en relation des projets, des stratégies, des visées, d'une part, et des cadres concrets à mettre en œuvre et à mettre à contribution.

Mobilisée dans une revue comme Quaderni, ancrée à la fois en Science politique et en Sciences de l'information et de la communication, la notion d'autorité illustre particulièrement bien comment un regard sur le pouvoir doit rencontrer une perspective sur les médiations communicationnelles des objets dans la société.

Or l'autorité ainsi approchée ne peut être conçue ni comme une qualité des acteurs, ni comme une substance objective qu'ils détiendraient : 
l'emprise des dispositifs, d'une part, et la prise des utilisateurs sur leurs fonctionnalités et leurs opportunités, d'autre part, composent l'autorité, ou plutôt les situations d'autorité, comme des phénomènes dynamiques à la rencontre de médiations hétérogènes. On a donc toujours affaire à des processus d'autorisation, qui sont le cœur du questionnement soulevé ici.

Comment, donc, les acteurs composent-ils leur autorité, en relation à la fois avec des ordres sociaux de légitimité et avec les cadres particuliers qu'offrent à ces dynamiques les médias en ligne ? Pour aborder une telle question, il nous a paru nécessaire de faire porter l'analyse sur trois niveaux différents.

\section{La notion d'autorité, avant et après le numérique}

Cette question est relative notamment à l'idée d'une « crise de l'autorité » dans le monde contemporain, du fait des transformations liées à l'autorité transcendantale traditionnelle et de l'émergence d'« autorités numériques ».

D'après les chercheurs contemporains, ce que Hannah Arendt a qualifié de crise d'autorité en 1968 ne serait que la transformation profonde de l'attitude sociale envers la figure de grand leader, une attitude qui engendrait auparavant "la croyance superstitieuse à une supériorité plus ou moins transcendante, héritée d'un Dieu obscur et arbitraire qui lui autorise une action hors de toute règle et sans contrôle d'une loi » (Damien, 2012, p. 24). Le besoin collectif de suivre des personnalités superlatives et charismatiques qui peuvent changer le cours de l'Histoire a été remplacé progressivement par l'intention de l'implication directe dans des processus de révélation d'autorité qui se veulent « collectifs », « démocratiques » et « transparents ».

La remise en question des autorités transcendantes coïncide souvent avec l'évolution et la grande diffusion des supports numériques qui permettent l'interaction continue et simultanée à distance. Ces supports qui semblent souvent mettre à égalité des acteurs de statuts et de catégories sociales différents transmettent le sentiment de la transparence via des processus de médiation technique. Dans ce cadre, Robert Damien parle de transformations « médiologiques » qui ont comme conséquence la mise en contact des différentes identités et cultures, matrices polymorphes de génération d'autorités. Ces médias numériques imposent leur méthodologie qui consiste en des procédures axiologiques basées sur la quantification. De nature calculatoire, le numérique est conforme à la représentation sociale du chiffre ; comme Étienne Candel le remarque, «dans le social, donner un chiffre, une mesure, une élaboration numérique de l'objet du discours, c'est apporter à la chose dite le caractère de l'exactitude, la validité supposée évidente, voire indiscutable, que porte un traitement statistique ou mathématique, une mesure » (2008, p. 38). Les acteurs en quête d'autorité mettent en place des pratiques qui leur apporteront plus de « clics », plus d'« amis », plus de « suiveurs », plus de « vues» ou plus de « liens entrants ». C'est par exemple un enjeu pour les marques, en quête d'une position culturelle au sein du monde social (Marti, 2015). Par ailleurs, ils doivent s'adapter aux particularités et aux contraintes de communication induites 
par le dispositif puisque leur participation doit respecter le type et la quantité des contenus mis en ligne, les types d'échange, le vocabulaire et le style d'expression des autres utilisateurs. Dans son article « Le processus de la légitimation des acteurs politiques en ligne. Exemple $\mathrm{du}$ discours des figures politiques françaises et russes sur Twitter », Tatiana Kondrashova pose de cette façon la question des pratiques d'autovalorisation des acteurs politiques au sein des dispositifs de réseautage social et questionne leur adoption des logiques médiatiques. Comment un acteur politique construit-il son image sur Twitter et s'adapte-t-il aux règles imposées par ce dispositif? Éric Sotto, quant à lui, s'interroge sur l'émergence des règles et des procédures qui imprègnent les pratiques conversationnelles des lecteurs dans un espace de discussion auto-géré : comment ce jeu d'autorisations, dans un contexte de participation libre sans modération, finit-il par faire émerger de nouvelles formes d'expertise ?

\section{$L^{\prime}$ « autorité » et son environnement notionnel}

Au fur et à mesure de l'évolution des technologies de communication numérique, plusieurs termes ont été utilisés pour décrire la présence et surtout l'influence des acteurs en ligne, et la notion d'autorité est travaillée, socialement, par ses relations avec des notions qui lui sont progressivement apparentées ou appariées : « crédibilité », « réputation », « visibilité, « légitimation», « reconnaissance », entre autres, viennent ainsi entourer et déterminer les modalités par lesquelles la notion d'autorité trouve aujourd'hui son sens « trivial » (Jeanneret, 2014) sur les réseaux. Pour les professionnels du web, la « visibilité » signifie par exemple une présence forte dans un univers de surabondance informationnelle. Dans la littérature sociologique, ce terme métaphorique issu de la météorologie, décrit le fait d'être reconnu dans une situation de reconnaissance dissymétrique où un petit nombre de gens crée un " capital de visibilité » (Heinich, 2012). Encore une fois l'aspect calculatoire est mis en avant. Plusieurs médias de réseautage socioprofessionnel sont basés sur une idée de " visibilité » qui traduit la reconnaissance capitalisée via des processus de quantification de l'approbation des autres participants (Gkouskou-Giannakou, 2016). Ainsi, au sein d'une « communauté » métaphorisée, la visibilité en tant que valeur scientifique devient un processus " démocratique » de " vote » et de «popularité » qui renforce le sentiment de « transparence ».

La « visilibilité » devient d'ailleurs un enjeu professionnel dans le cadre de la recherche par mot-clé ou du référencement des sites web par les moteurs de recherche. Pour Dominique Cardon, l'influence mesurée selon des méthodes calculatoires dans le web est une "autorité des méritants ». Cette autorité résulte des techniques de référencement des moteurs de recherche basées sur « les jugements humains émis par l'ensemble des internautes qui publient sur le web» (Cardon, 2015, p.26). De la même façon, la «popularité » est désignée par le nombre de consultations d'un contenu et la « réputation » par le nombre d'informations « partagées » et « commentées » dans les médias de réseautage social.

Une autre notion-phare mise en avant tant par les professionnels du web que par les chercheurs est celle de « crédibilité ». La notion de crédibilité est reliée à celle de persuasion comme capacité 
de conquérir la confiance d'autrui. Cette capacité peut être attribuée à une personne, à un support, à un média, à une institution ou elle peut concerner le contenu ou des caractéristiques extrinsèques du locuteur (pouvoir, popularité...). Or, d'après Alexandre Serres, la notion de web credibility « déborde la crédibilité du support et du média, pour englober tous les composants des documents : source, message, présentation » (Serres, 2012). Quant à B. J. Fogg, il distingue quatre types de crédibilité sur le web : la crédibilité « présumée », basée sur la notoriété, la crédibilité « réputée » fondée sur les avis, la crédibilité « gagnée » basée sur le contact et l'expérience vécue et l'expérience « superficielle » qui repose sur le design d'un site web.

Les notions de crédibilité, de réputation ou de popularité imprègnent celle d'autorité. Dans ce dossier, Camille Alloing parle d' " autorité réputationnelle » et d' "indicateurs de réputation numérique » en essayant de décrire les méthodes qui sont mises en œuvre par les usagers pour l'identification des sources d'information paraissant légitimes. De son côté, Aurélia Lamy analyse la notion d'autorité sous l'angle de la « confiance » envers les professionnels de la santé et de la légitimité sur les forums de discussion de patients en ligne.

\section{La construction discursive de l'autorité sur le web}

Ancrée dans une prise en compte du contexte social et des médiations matérielles de la communication, la problématique de la construction discursive de l'autorité fait ressurgir la question de la performativité du discours. Dans Ce que parler veut dire, Pierre Bourdieu (1982) considérait le discours comme porteur d'une autorité extrinsèque. En séparant le système économique et social du système linguistique, il relevait dans ce dernier l'obligation de s'adapter à son contexte afin de se légitimer. Or, l'attribution de valeur et de légitimité à une langue ou à un mode d'expression, «c'est-à-dire de la reconnaissance qui lui est accordée » (p. 47) est toujours extérieure. Les règles de légitimation d'une écriture sont posées par «les écrivains, auteurs plus ou moins autorisés» et par «les grammairiens, détenteurs du monopole de la consécration et de la canonisation des écrivains et des écritures légitimes » (p. 48). Ensuite, ces règles se normalisent et se diffusent via les institutions scolaires.

Pour Michèle Monte et Claire Oger, le discours peut être considéré comme un élément constitutif de l'autorité ; en distinguant le « discours d'autorité » du "discours autoritaire », ces auteurs ont mis en valeur les phénomènes d'inscription du premier dans des «matérialités discursives » (2015, p. 7). Dans leur perspective, l'autorité émerge d'un summum de crédibilité et d'argumentation ; ainsi, la crise d'autorité évoquée par Hannah Arendt résulterait de l'absence d'autorité intrinsèque dans un discours institutionnel.

À l'ère de l'Internet les formes des discours sont souvent formatées par le dispositif ; en d'autres termes, le dispositif qui autorise l'énonciation en règle aussi les modalités. Pour Gloria Origgi (2015), le discours humain sur Internet est en partie délégué aux logiciels qui gèrent son formatage et sa diffusion. Ces mêmes logiciels gèrent aussi l'autorité, la confiance et la réputation du contenu de ce discours. L'auteur examine notam- 
ment d'un œil critique les dispositifs numériques de communication académique qui induisent un discours d'auto-citation dans un contexte de calcul de productivité scientifique.

Dans l'univers de l'écriture journalistique, les exemples d'autorité discursive intrinsèque sont particulièrement nombreux autant avant qu'après la diffusion du numérique. Si, auparavant, l'autorité du discours des médias journalistiques se situait dans leur rhétorique comme capacité de convaincre le public, aujourd'hui cette autorité est souvent basée sur le paradoxe d'une écriture qui s'appuie sur l'égalité (à travers des pratiques comme le crowdsourcing), le direct (à travers le «partage » des actualités dans les réseaux sociaux) ou l'illusion de la véracité des statistiques, comme par exemple dans le cas du « journalisme de données » (Gkouskou-Giannakou, 2015).

Dans ce dossier, Marion Rollandin analyse la notion d'autorité comme un processus d'autorisation personnelle et collective dans les espaces médiatisés participatifs. Dans son article, elle décrit les méthodes de construction d'autorité mises en œuvre par les acteurs dans les dispositifs de communication en ligne. Pour leur part, Ariane Mayer et Nicolas Sauret s'interrogent sur la dualité « autorité/auteur » à partir de l'étude d'un espace de fiction transmédia collaboratif. Ils observent trois types d'autorité (topologique, narrative, dispositive) qui émergent à travers l'interaction littéraire.

En somme, l'autorité observée ici est moins le fait d'institutions et de légitimités proprement politiques que la forme d'un pouvoir plus diffus, un soft power en quelque sorte, où le projet de communication, son cadre matériel dans le dispositif et son contexte social se déploient et opèrent un certain pouvoir sur le public des lecteurs et usagers des médias informatisés. Ce dossier interroge la façon dont la manipulation des formes numériques permet aux acteurs de s'autoriser, de se légitimer, de se faire valoir dans un projet personnel ou collectif, de se qualifier dans une expertise, un savoir ou une écriture. 


\section{$R \cdot E \cdot F \cdot E \cdot R \cdot E \cdot N \cdot C \cdot E \cdot S$}

ARENDT Hannah, «Qu'est-ce que l'autorité ? », La crise de la culture, Paris, Gallimard, 1972. BOURDIEU Pierre, Ce que parler veut dire: l'économie des échanges linguistiques, Paris, Fayard, 1982.

CANDEL Étienne, «Une ration quotidienne de statistiques. La pratique éditoriale du «chiffre du jour» dans la presse écrite », in $M E I, \mathrm{n}^{\circ} 28$, «La communication nombre », 2008, pp. 37-53. CARDON Dominique, À quoi rêvent les algorithmes?, Paris, Le Seuil, 2005.

DAMIEN Robert, Éloge de l'autorité : généalogie d'une (dé)raison politique, Paris, Armand Colin, 2013.

DE MUNCK Jean, "Les métamorphoses de l'autorité ", in Autrement, n 198, 2000, pp. 21-42.

FOGG B. J., Persuasive Technology: Using Computers to Change What We Think and Do, San Francisco, Morgan Kaufmann, 2003.

GKOUSKOU-GIANNAKOU Pergia, "Sites de réseautage social et autorité scientifique. Le cas de Researchgate ", in Les écosystèmes numériques. Intelligence collective, Développement durable, Interculturalité, Transfert de connaissances, Presses des Mines, Paris, 2016, pp. 215-228.

GKOUSKOU-GIANNAKOU Pergia, «Transformation des autorités médiatiques et lien social. À propos des formes d'écriture «démocratisées» du journalisme de données ", in Nouer le lien social. Pratiques de communication et lien social, éditions UBP, 2015, pp. 139-160.

GOMEZ-MEJIA Gustavo, Les fabriques de soi ? Identité et industrie sur le Web, Paris, MkF éditions, 2016.

HEINICH Nathalie, De la visibilité. Excellence et singularité en régime médiatique, Paris, Gallimard, 2014.

JEANNERET Yves, Critique de la trivialité : les médiations de la communication, enjeu de pouvoir. Paris, Éditions Non Standard, 2014.

LICKLIDER Joseph, TAYLOR Robert, «The Computer as a Communication Device ", in Science and Technology, 1968, p. 21-41.

MARTI Caroline, "Les médiations culturelles des marques : une quête d'autorité », ouvrage inédit, in De la gestion sémiotique à la prétention sociale des marques. Une analyse communicationnelle des pratiques du marketing, mémoire pour l'Habilitation à diriger des recherches, sous la direction de Yves Jeanneret, GRIPIC, Celsa Paris-Sorbonne, 2015.

MONTE Michèle, OGER Claire, « La construction de l'autorité en contexte. L'effacement du dissensus dans les discours institutionnels ", in Mots. Les langages du politique, $\mathrm{n}^{\circ} 107,2015$, pp. 5-16.

ORIGGI Gloria, La réputation. Qui dit quoi de qui, 2015, Paris, PUF.

RHEINGOLD Howard, Les communautés virtuelles, Paris, Addison-Wesley France, 1993.

SERRES Alexandre, Dans le labyrinthe, Paris, C\&F éditions, 2012. 
